\title{
Balding Black Holes Lose Their Magnetic Hair
}

\author{
First-principles plasma simulations show that black holes can't keep their \\ magnetic fields. \\ By Rachel Berkowitz
}

A ccording to the physicist John Wheeler, "black holes have no hair"--they can be described entirely by their

mass, charge, and spin. However, some researchers have proposed that a classical black hole surrounded by a plasma could retain "hair" in the form of a magnetic field. Ashley Bransgrove, at Columbia University, and his colleagues now show that such a black hole does satisfy the no-hair theorem after all [1]. The result could be tested by $x$-ray and infrared observations of supermassive black holes, like the one at the center of our Galaxy.

A black hole may be born with a magnetic field, or it may acquire one by swallowing magnetized material. The no-hair theorem says that in such cases, the magnetic field on the event horizon of an isolated black hole should be quickly radiated away, returning the black hole to a "bald" state. But astrophysical black holes don't exist in isolation. Rather, they are surrounded by plasma that prevents the magnetic field from sliding off the event horizon. What does this mean for the no-hair theorem?

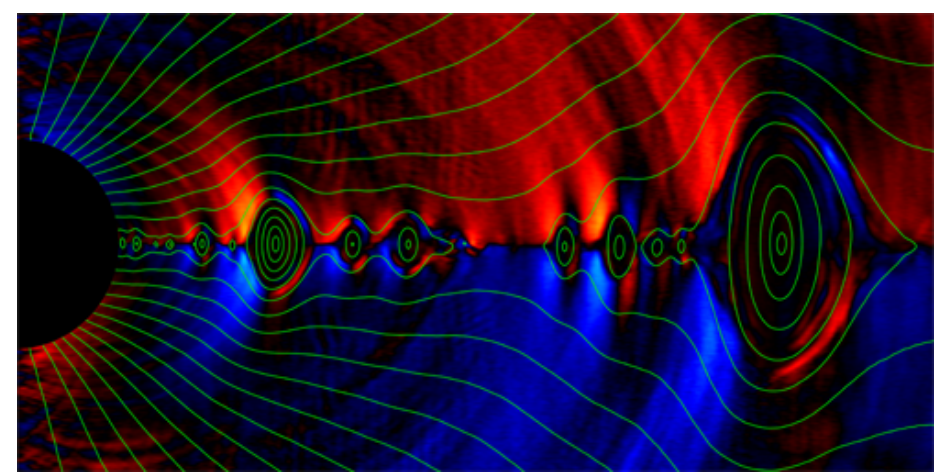

To find out, Bransgrove and his colleagues simulated a magnetic field around a black hole in the presence of collisionless and collisional plasmas. They found that the magnetic field lines break, reconnect, and form plasma-filled magnetic loops that either escape into space or fall into the black hole. The faster the reconnection rate, the quicker the magnetic field decays and the sooner the no-hair theorem is satisfied.

In strong magnetic fields, reconnection produces high-energy $x$ rays. This means that, as well as keeping black holes hair-free, the process could explain observations of $x$-ray flares and hotspots near supermassive black holes.

Rachel Berkowitz is a Corresponding Editor for Physics based in Vancouver, Canada.

\section{REFERENCES}

1. A. Bransgrove et al., "Magnetic hair and reconnection in black hole magnetospheres," Phys. Rev. Lett. 127, 055101 (2021).

Credit: A. Bransgrove et al. [1] 